\title{
Editor Note
}

\section{Kelly Stefano Cole}

Associate Professor of Immunology, Center for Vaccine Research, University of Pittsburgh, Pittsburgh, PA, USA

*Corresponding author: Kelly Stefano Cole, PhD, Associate Professor of Immunology, Center for Vaccine Research, Pittsburgh, PA, USA, E-mail: stefcole@pitt.edu Received date: Aug 01, 2016; Accepted date: Aug 02, 2016; Published date: Aug 05, 2016

Copyright: (C) 2016 Cole KS, This is an open-access article distributed under the terms of the Creative Commons Attribution License, which permits unrestricted use, distribution, and reproduction in any medium, provided the original author and source are credited.

\section{Editor note}

Vaccine mimic the disease causing organism in order to provide acquired immunity to the living organism. Emerging practices and experiences in this field needs a comprehensive discussion to benefit those in need of protective immunity to emerging infections.

Journal of Vaccines \& Vaccination Volume 7, Issue 3 comprises original research articles evaluating vaccine strategies for endemic infectious diseases in several countries around the world.

Russian author Kiseleva et al., presented the the Stability of attenuating mutations as an important evidence for the live attenuated influenza vaccine (LAIV). This study describes Phase I clinical trials of three Russian LAIVs against potentially pandemic influenza viruses, which may cause serious and fatal disease in humans.

Evaluated the implementation platform the modified vaccinia virus Ankara (MVA). Researchers presented data from a clinical trial 32 KOL's (key opinion leaders) representing the regulatory, industry, and academia fields. They deployed quantitative techniques for analysis through various ranking methods. The study states regulatory, industry, and academia must find a common ground perspective and collaborate to overcome regulatory and social barriers to vaccination, and promote strategic consensus for implementation of this vaccination strategy.

Reverse Vaccinology allows rapid and efficient ways to analyze putative immunogenic antigens. Singh et al. have established reverse vaccinology as a powerful tool to identify vaccine candidates. Studies have revealed that outer membrane proteins of A. baumannii could serve as vaccine candidates or effective treatment options against baumannii MDRA. The present study reported NucAb as an outer membrane nuclease in silico a one such vaccine candidate survival by $20 \%$ in active immunization and $40 \%$ survival on passive immunization.
The study from the authors (Sugun et. al.) problem on prevalence of P. multocida, an aetiologic agent of bovine hemorrhagic septicemia, in West Africa. Specifically, his group reports. About P. multocida capsular and somatic strains it in the entire West African sub-region in general and Nigeria in particular. The study emphasizes the need to redefine the vaccine strategies for and recommends further research in other parts of the country to gather more relevant information with regards to capsular and somatic type of P. multocida.

Nosocomial infections such as Clostridium difficile are a global issue in hospitals around the world, and account for billions of dollars in healthcare costs annually. Maldarelli et al. present data from animal models demonstrating that simple vaccination of mice with C. difficile pilins lead to generation of antibodies that protect against C. difficile challenges in vivo. Authors hypothesize that human vaccinations such as these may serve to reduce the healthcare burden annually, and should be evaluated. This article hypothesized that vaccinating mice with pilins would lead to generation of anti-pilin antibodies, and would protect against $\mathrm{C}$. difficile challenges.

Goh et al. reports on Non-typhoidal Salmonella (NTS) disease as a major public health burden, In light of limited research to date, the present study provides proof-of-principle evidence that targeting flagella with antibodies can increase the antibacterial function of host cells, with IgG3 being the most potent subclass. The study emphasized the need for optimized vaccines against iNTS diseases.

Denmark researchers Mortensen et al. demonstrated emerging issue on New Generation Combination Vaccines against Streptococcus pyogenes. This study characterizes GAS (Spell out) -based vaccines, demonstrating their ability to induce Th1/Th17/IgG3 based memory immunity in humans.

"Combined, these articles demonstrate the need to evaluate a variety of countries that include diversity of geography, ethnicity, socioeconomic, and other backgrounds. 